\title{
Electrical Switching in Thin Film Structures Based on Molybdenum Oxides
}

\author{
A. L. Pergament, V. P. Malinenko, L. A. Aleshina, E. L. Kazakova, and N. A. Kuldin
}

Petrozavodsk State University, Petrozavodsk 185910, Russia

Correspondence should be addressed to A. L. Pergament; aperg@psu.karelia.ru

Received 21 May 2014; Revised 21 August 2014; Accepted 2 September 2014; Published 18 September 2014

Academic Editor: Tatyana Sizyuk

Copyright @ 2014 A. L. Pergament et al. This is an open access article distributed under the Creative Commons Attribution License, which permits unrestricted use, distribution, and reproduction in any medium, provided the original work is properly cited.

\begin{abstract}
We report on the experimental study of electrical instabilities in thin film structures on the basis of molybdenum oxides. Thin films of molybdenum oxide are obtained by thermal vacuum evaporation and anodic oxidation. The results of X-ray structural analysis, investigation of optical and electrical properties, are presented. It is shown that the initial vacuum-deposited oxide represents amorphous $\mathrm{MoO}_{3}$. In the $\mathrm{MOM}$ (metal-oxide-metal) structures with Mo oxide films obtained by the two methods, the effect of electrical switching with an S-shaped current-voltage characteristic is found. We put forward a hypothesis according to which the switching mechanism is associated with the development of electrical instability caused by the insulator-to-metal transition in $\mathrm{Mo}_{8} \mathrm{O}_{23}$. The switching channel, comprising this lower valence oxide, emerges in the initial film during the process of electrical forming of the MOM structure. The obtained results indicate the possibility of application of these structures in oxide micro- and nanoelectronics as electronic switches and other electronic devices.
\end{abstract}

\section{Introduction}

Transition metal oxides (TMO) represent one of the most promising classes of substances with regard to their use in designing electronic components [1]. Molybdenum-oxygen system, like most TMOs, forms a number of oxides with variable valence of the cation, which are characterized by a variety of structural and physicochemical properties [1-3]. The basic binary molybdenum oxides are $\mathrm{MoO}_{2}$ and $\mathrm{MoO}_{3}$.

Molybdenum dioxide crystallizes in the monoclinic structure which is formed by infinite chains of distorted octahedra $\mathrm{MoO}_{6}$, faces connected to each other due to van der Waals forces. Molybdenum trioxide $\mathrm{MoO}_{3}$ has a layered structure, whose main element is a double octahedron with a $\mathrm{MoO}_{6}$ link [2-5]. Molybdenum trioxide has several polymorphs, and the most thermodynamically stable of them is $\alpha-\mathrm{MoO}_{3}$ (space group Pnma). By lowering the ratio of oxygen to metal content down to 2.9 and below, one of the seven stable or metastable suboxides can form: $\mathrm{Mo}_{18} \mathrm{O}_{52}$, $\mathrm{Mo}_{9} \mathrm{O}_{26}, \mathrm{Mo}_{8} \mathrm{O}_{23}, \mathrm{Mo}_{5} \mathrm{O}_{14}$, and $\mathrm{Mo}_{17} \mathrm{O}_{47}$, as well as $\eta$ - and $\gamma-\mathrm{Mo}_{4} \mathrm{O}_{11}$ [3].

Stoichiometric $\mathrm{MoO}_{3}$ is an insulator with a band gap of $\sim 3 \mathrm{eV}$ [1]. During reduction by means of either oxygen deficiency formation or introducing donor impurity atoms, additional donor levels near the conduction band bottom appear and the reduced oxide behaves therefore as a semiconductor. Polycrystalline $\mathrm{MoO}_{3}$, as well as a single crystal, has a gray-yellow color and the minimum value of the absorption edge is $440 \mathrm{~nm}$ which corresponds to the smallest value of the band gap of $2.8 \mathrm{eV}$ [6]; thus, the band gap depends upon the stoichiometry and the crystalline state. The conductivity values of molybdenum oxides range from dielectric $\mathrm{MoO}_{3}$ to semiconductor $\mathrm{Mo}_{18} \mathrm{O}_{52}$ (resistivity $\rho=78.1 \Omega \cdot \mathrm{cm}$ ) and down to metal $\eta-\mathrm{Mo}_{4} \mathrm{O}_{11}\left(\rho=1.66 \times 10^{-4} \Omega \cdot \mathrm{cm}\right)$ [6]. Molybdenum dioxide also exhibits metallic properties $[1,6]$ (for bulk samples $\rho=8.8 \times 10^{-5} \Omega \cdot \mathrm{cm}$ at room temperature [2]).

Due to its special physical and chemical properties, molybdenum oxides may find applications in electronic devices, such as electrochromic displays and indicators, reversible cathodes of lithium batteries, gas sensors, and memory elements $[2,3]$. For instance, the effects of resistive switching memory in structures based on oxides of Mo have been observed in a number of studies $[7,8]$, and the phenomena of photochromism and electrochromism in $\mathrm{MoO}_{3}$ have been described in [9-11]. 
Metal-insulator transitions (MIT) have also been observed in some oxides of molybdenum and related compounds. In particular, $\mathrm{Mo}_{8} \mathrm{O}_{23}$ exhibits a two-stage structural MIT with formation of a charge-density wave (CDW) at $T_{c 1}=315 \mathrm{~K}$ and $T_{c 2}=285 \mathrm{~K}[12]$, and $\eta-\mathrm{Mo}_{4} \mathrm{O}_{11}$ demonstrates similar transitions at temperatures, respectively, 109 and $35 \mathrm{~K}$ [13]. The Mott MIT has been reported in $\mathrm{R}_{2} \mathrm{Mo}_{2} \mathrm{O}_{7}$ (where $\mathrm{R}$ is a rare earth element) [14] and the Peierls transition-in quasi-1D potassium molybdenum bronze $\mathrm{K}_{0.3} \mathrm{MoO}_{3}$ [15]. An electron-induced MIT has been supposed to occur in quasi2D single-layer molybdenum disulfide [16], and resistive switching memory in $\mathrm{MoS}_{2}$ nanosphere assemblies has been reported in [17]. In the work [18], a MIT at $T_{c}=380 \mathrm{~K}$ has been found in hydrogen bronze $\mathrm{H}_{0.33} \mathrm{MoO}_{3}$ associated with the ordering of $\mathrm{H}$ atoms, unlike bronzes $\mathrm{A}_{x} \mathrm{MoO}_{3}$ (where $\mathrm{A}$ is an alkali metal) and lower molybdenum oxides (i.e., the above-mentioned $\mathrm{Mo}_{4} \mathrm{O}_{11}$ and $\mathrm{Mo}_{8} \mathrm{O}_{23}$ ) in which the low-temperature Peierls transition is due to the instability of the Fermi surface and the formation of CDWs [19]. In addition, it has been reported [20] on the inverse (when the low temperature phase is metallic) MIT in the glasses of $\mathrm{MoO}_{3}-\mathrm{TeO}_{2}$ composition, at $T_{c}$ in the range of $230-340 \mathrm{~K}$, depending on the molybdenum oxide concentration.

Note that memory switching is observed in many TMOs $[8,21,22]$. The most discussed models in the literature for the ReRAM mechanism in oxide structures are those based either on the growth and rupture of a metal filament inside the oxide matrix under the action of electric current or on the redox processes responsible for the formation of some high-conductivity or low-conductivity local inclusions corresponding to a particular oxygen stoichiometry [22]. The MIT ideology is also sometimes involved to explain the properties of the structures and the memory switching mechanism therein [23]. In any case, the memory switching phenomenon seems to be associated with the ion transport $[22,24]$. It is also appropriate to mention here the works discussing the memory effects in a material with MIT (vanadium dioxide) associated with the presence of hysteresis in the temperature dependence of conductivity [25].

On the other hand, monostable threshold switching (with no memory effects) associated with the MIT is also characteristic of a number of TMOs $[22,26,27]$. That is why the study of threshold switching in molybdenum oxide and its possible connection with the phenomenon of MIT is of considerable scientific and practical interest. On the basis of the aforesaid, the purpose of this work is to study the electrical properties of thin-film MOM ("metal-oxide-metal") structures based on molybdenum oxide and to identify the basic regularities of the effects of electrical forming and switching in these structures.

\section{Sample Preparation and Experimental Methods}

Thin film samples of molybdenum oxide were obtained by two ways: anodic oxidation [28] and thermal vacuum evaporation.

Anodization of molybdenum was carried out in an acetone-based electrolyte (22 $\mathrm{g}$ of benzoic acid plus $40 \mathrm{~mL}$ of saturated borax aqueous solution per liter of acetone) $[27,29]$. Precleaning of the surface of the molybdenum samples was produced by chemical etching in a $5 \%$ aqueous solution of hydrochloric acid followed by washing in distilled water and drying in hot air stream. Immediately after anodizing, the oxide was very sensitive to water and water vapor. Furthermore, after extracting the sample from the electrochemical cell, electrolyte residues, due to rapid acetone evaporation, aggressively exposed the as-fabricated oxide film to form an inhomogeneous surface. Therefore, after oxidation in the electrolyte, the samples were carefully washed with pure acetone and dried instantly. Anodic oxidation was carried out in galvanostatic regime; the current was $I_{a}=$ $70 \mathrm{~mA}$ (with oxidized surface area of $S \sim 1 \mathrm{~cm}^{2}$ ) and the oxidation time was $t=5$ to 10 minutes. The voltage drop $V_{a}$ across the electrochemical cell increased to values of $30-35 \mathrm{~V}$ in the first few tens of seconds and then reached saturation. This unusual behavior of $V_{a}$ (no linear growth) and relatively high values of $I_{a}$ could be due to the high electronic conductivity of the product of oxidation (which might include lower molybdenum oxides, e.g., $\mathrm{Mo}_{4} \mathrm{O}_{11}$ and $\mathrm{MoO}_{2}$, with a relatively high electronic conductivity) and the possible dissolution of the film. The fact that the anodic oxide on molybdenum contained lower oxides was confirmed by experimental data [30], where it had been shown that the degree of oxidation (valence state) of molybdenum in an anodic oxide film (AOF) is less than six. Also, this was confirmed theoretically in terms of the thermodynamics of the process: the formation of unsaturated oxide $\mathrm{MoO}_{2}$ had been shown to be energetically more favorable than $\mathrm{MoO}_{3}$ [31].

Vacuum deposited film samples were fabricated by thermal evaporation of $\mathrm{MoO}_{3}$ powder at residual pressure of $10^{-4}$ Torr onto polished tantalum plates or glass substrates, for electrical and optical studies, respectively, using a vacuum universal post VUP-5 M. A special evaporator (covered boat with holes) made of $0.2 \mathrm{~mm}$ thick tantalum foil was used. All substrates were subjected to a pretreatment in alcohol, after which they were washed in distilled water and dried, and the final heat treatment was carried out directly inside the working vacuum chamber of VUP-5 M, where they were heated to a temperature of $400^{\circ} \mathrm{C}$. The thickness of the deposited layers was determined from the transmission and reflection interference spectra [32] and was found to be 300 to $800 \mathrm{~nm}$. Unlike AOFs, vacuum deposited films were stable against atmosphere water.

Current-voltage characteristics of the samples were measured in sandwich geometry structures with the springloaded top electrode ( $\mathrm{Au}$ wire $0.5 \mathrm{~mm}$ in diameter), and the bottom electrode was either molybdenum (in the case of anodic oxide) or tantalum metal. Samples were subjected to preliminary electroforming at an $\mathrm{AC}$ voltage, close to the breakdown voltage, for a certain time. The electroforming process and $I-V$ curves after forming were investigated by the oscillographic method [27] (Figure 1) in the AC dynamic mode using a digital oscilloscope OWON PDS 50226 with filing the data to a PC. All the electrical measurements were 
conducted in air at standard conditions (room temperature, atmospheric pressure, and humidity; also, in some cases, the temperature was varied from $\mathrm{RT}$ to $100^{\circ} \mathrm{C}$ ).

$\mathrm{X}$-ray studies were made with a DRON-6 diffractometer using $\mathrm{Fe} \mathrm{K}_{\alpha}$ radiation, and measurements of the reflectance and transmittance spectra were carried out using an SF-56 spectrophotometer in the wavelength range $200-1100 \mathrm{~nm}$.

\section{Experimental Results and Discussion}

X-ray diffraction pattern of a vacuum-deposited molybdenum oxide sample is presented in Figure 2(a). The study of phase composition and structure shows that the samples are amorphous $\mathrm{MoO}_{3}$ with a slight oxygen nonstoichiometry [4].

Anodic oxide films on Mo, just as those obtained by vacuum deposition, are amorphous (or amorphousmicrocrystalline, according to the literature data [29]), but their composition corresponds, as described above in Section 2, rather to $\mathrm{MoO}_{2}$ and not to the highest oxide. Figure 2(b) shows the reflection and transmission spectra for one of the samples. The experimental data processing using the envelope of the interference extrema [32] yields the film thickness of $(620 \pm 50) \mathrm{nm}$, and the absorption edge around $\lambda \approx 400 \mathrm{~nm}$ indicates the fact that the oxide phase composition corresponds to $\mathrm{MoO}_{3}$ with a band gap of $\sim 3 \mathrm{eV}$.

Initial structures demonstrate the nonlinear and slightly asymmetric current-voltage characteristics with no regions of negative differential resistance (NDR). When the amplitude of the applied voltage reaches the forming voltage, a sharp and irreversible increase in conductivity is observed and the $I(V)$ curve becomes S-shaped (Figure 3). With increasing current, the $I-V$ characteristic may change until the parameters of the switching structure are finally stabilized. The process outlined above is qualitatively similar to the electroforming of the switching devices based on other TMOs [22, 27].

Thus, the first stage of the forming does not differ from the conventional electrical breakdown of oxide films. However, if the postbreakdown current is limited (by means of sufficiently high value of $R_{L}$; see Figure 1), this results in formation of a switching channel rather than a breakdown one. The latter would be expected to have metallic-like conductivity and no negative resistance in the $I-V$ characteristic. It is quite evident that the phase composition of this switching channel must differ from the material of the initial oxide film, because the channel conductivity exceeds that of an unformed structure by several orders of magnitude.

After electroforming, the $I-V$ characteristics, as was already said, are S-shaped (Figure 3). The samples are initially in a high resistance (OFF) state; when the applied voltage reaches the threshold value $V_{\text {th }}$, a sharp increase in conductivity is observed and the sample switches into a low resistance $(\mathrm{ON})$ state. The described behavior is completely reversible, though at the forward and backward voltage sweep, the $I-V$ curves demonstrate a slight hysteresis in the NDR region.

The $I-V$ curves for the evaporated and anodic films are qualitatively similar, but there is a significant quantitative difference in their threshold parameters. For instance, the switching voltage $V_{\text {th }}$ for the AOF based structures is $\sim 2 \mathrm{~V}$ (Figure 3(b)), whereas for the structures on the basis of

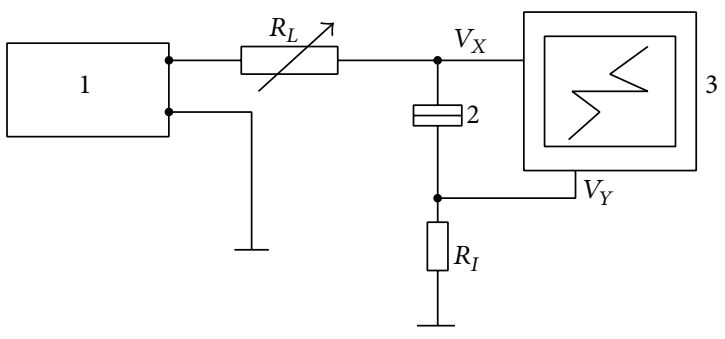

FIGURE 1: Experimental setup for $I-V$ measurements: 1: AC generator (frequency is $100 \mathrm{~Hz}$ ), 2: sample, 3: oscilloscope. $R_{L}$ is a load resistor and $R_{I}$ is a current-measuring resistor whose resistance is much less than the sample resistance.

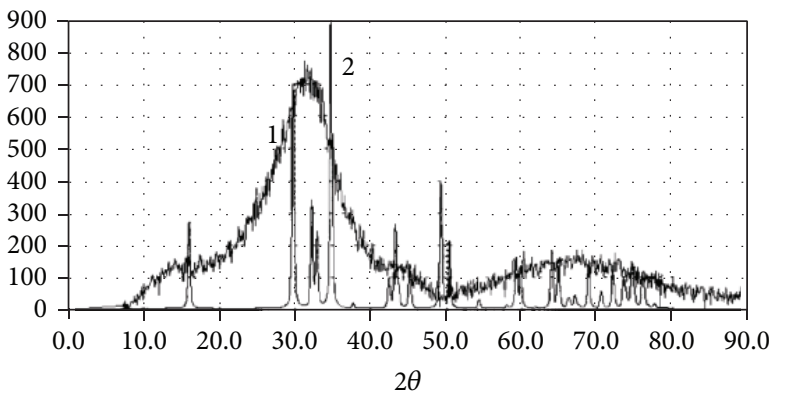

(a)

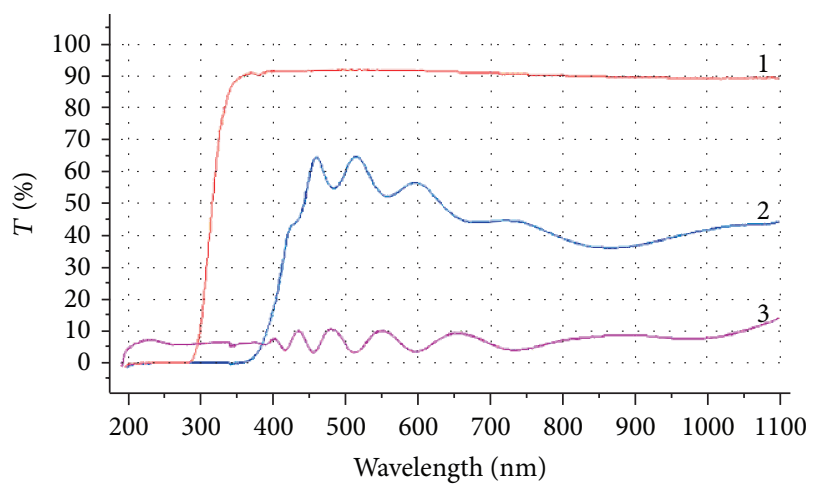

(b)

FIGURE 2: (a) XRD pattern of molybdenum oxide (1) compared with standard (2) $\mathrm{MoO}_{3}$ powder. (b) Optical spectra of the sample obtained by vacuum deposition: (1) transmission coefficient of substrate (glass); (2) transmittance; and (3) reflectance of Mo oxide film on glass [4].

the films prepared by thermal evaporation, $V_{\text {th }}$ is $\sim 10 \mathrm{~V}$ (Figure 3(a)). This difference can be explained by the difference in thickness of the films. Because of the heterogeneity of the thickness of AOFs (as can be judged by the heterogeneity of the interference color), their thickness has not been measured. However, the maximum (assuming the absolute efficiency of the reaction of anodic oxidation) thickness can be estimated from the Faraday's law [28]:

$$
d=\frac{\mu I_{a} t}{4 e \rho S N_{A}},
$$




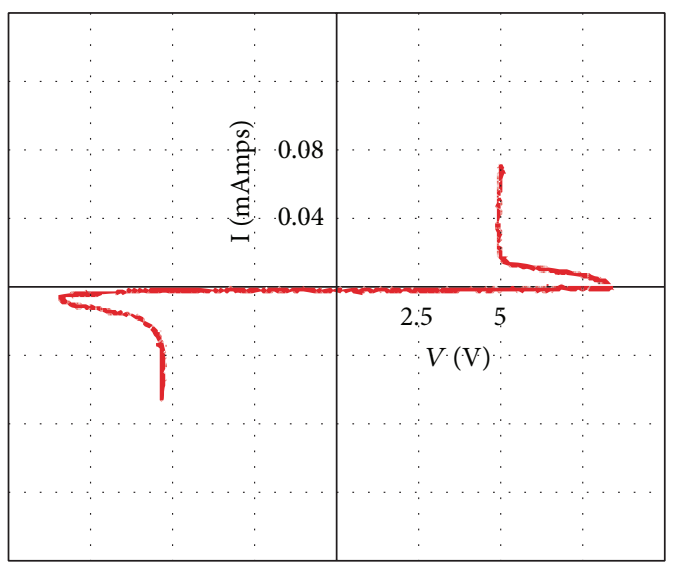

(a)

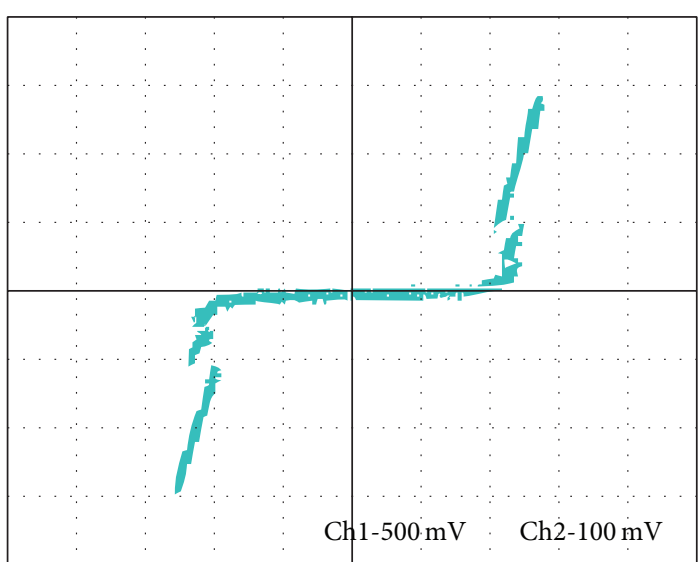

(b)

FIGURE 3: Current-voltage characteristics of MOM structures with vacuum-deposited (a) and anodic (b) Mo oxide films after electroforming. (b): X-0.72 V/div., Y-0.16 mA/div.

where $\mu=128 \mathrm{~g} / \mathrm{mol}$ and $\rho=6.5 \mathrm{~g} / \mathrm{cm}^{3}$ are, respectively, the molar mass and density of $\mathrm{MoO}_{2}$ and $e$ and $N_{A}$ - the unit charge and Avogadro constant. For $I_{a}=70 \mathrm{~mA}$ and $t=$ $10 \mathrm{~min}$., (1) yields $d \sim 20 \mathrm{~nm}$ which is significantly less than the typical thickness of the vacuum deposited films and, as a result, the threshold voltage for AOFs is also lower than that for the films obtained by thermal evaporation.

It should be noted that switching is not observed in thinner films obtained by vacuum evaporation $(d<100 \mathrm{~nm})$ in contrast to ultrathin AOFs. A plausible reason for this might be the fact that relatively thin vacuum deposited films contain through-the-thickness conducting defects due to local stoichiometry variations, whereas such defects are impossible in anodic films because they are grown in high electric field during anodic oxidation, and an appearance of such defects would interrupt the film growth process.

Next we note that, unlike the most other TMOs [22, 27], the process of electroforming in Mo-based structures was hindered, similarly to that in structures based on manganese and yttrium oxides $[33,34]$; that is, the conventional electrical breakdown often occurred, not the formation of a switching channel. However, for the Mo oxide films prepared by vacuum deposition, the processes of electroforming and switching were more stable which allowed in this case observation of the transformation of $I-V$ curves at a temperature change. It was found that, as the temperature increased, the threshold voltage was reduced. At $T=T_{0} \sim 55-65^{\circ} \mathrm{C}$ the NDR region degeneration commenced, at $T \approx 67^{\circ} \mathrm{C} V_{\text {th }} \rightarrow 0$, and at still higher temperatures, the switching effect no longer occurred (Figure 4).

It is known that during electroforming, a switching channel is formed under the electrode [27] due to crystallization and partial reduction of the initial oxide. In the case of electroforming of the structures based on $\mathrm{V}, \mathrm{Nb}, \mathrm{Ti}$, and Fe oxides, the most energetically favorable (in terms of a minimum Gibbs free energy $\Delta G_{r}$ in the corresponding reactions of the highest oxide reduction) is the formation of the channels consisting of $\mathrm{VO}_{2}, \mathrm{Ti}_{2} \mathrm{O}_{3}, \mathrm{NbO}_{2}$, and $\mathrm{Fe}_{3} \mathrm{O}_{4}$

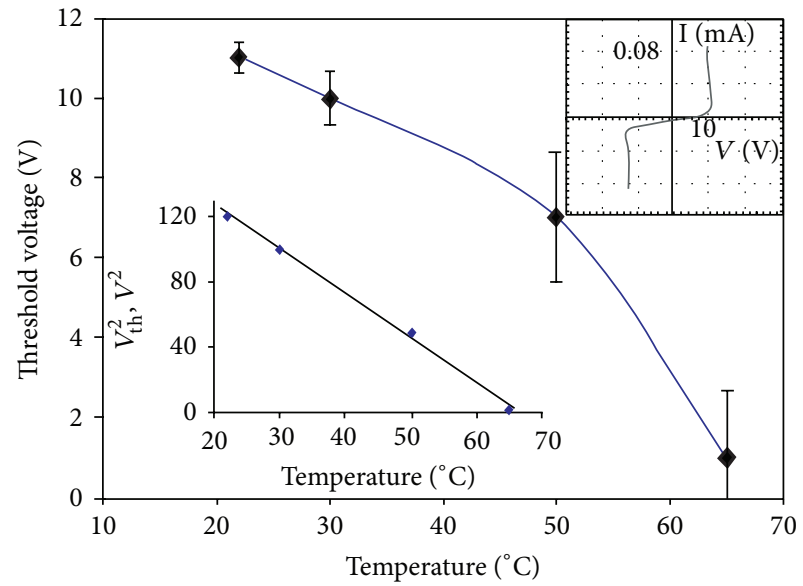

FIgURE 4: Threshold voltage as a function of temperature for the Ta$\mathrm{MoO}_{x}$-Au switching structure and the temperature dependence of $\left(V_{\mathrm{th}}\right)^{2}$ (lower inset) in compliance with (2). I-V curve at RT (upper inset).

[27], and a common feature for all these compounds is the MIT in them. At that, the threshold voltage decreases with increasing temperature tending to zero at $T$ corresponding to the transition temperature $T_{c}$ for each of these compounds.

In this case, the switching mechanism might be described in terms of the "critical temperature" model [35]. Due to the effect of Joule heating, when the voltage reaches a critical value $V=V_{\text {th }}$, the channel is heated up to $T=T_{c}$ and the structure undergoes a transition from the insulating OFF state to the metallic ON state. This is exactly the model of "critical temperature" (i.e., a simple electrothermal mechanism, albeit taking into account the specific $\sigma(T)$ dependence of the material at the MIT), though the mechanism of the MIT itself is, of course, essentially electronic. In this model

$$
V_{\text {th }} \sim\left(T-T_{c}\right)^{1 / 2} ;
$$


that is, the squared threshold voltage linearly tends to zero at $T \rightarrow T_{c}$ (see Figure 4, inset). Note that the value of $T_{c}$ in (2) does not coincide exactly with equilibrium transition temperature for the MIT in a particular material: for example, for vanadium dioxide this $T_{c}$ in (2) may vary in the range 310$330 \mathrm{~K}$, whereas the temperature of the MIT, in the absence of electric field, is equal to $340 \mathrm{~K}$ in this compound $[1,22,27,31$, 35].

One can surmise that in the case of Mo, formation of a channel also takes place, and this channel should consist of a material exhibiting MIT, which is the reason for switching with an S-shaped current-voltage characteristic (Figure 3).

A brief review of the materials with MIT in the family of molybdenum compounds (see Section 1) shows that the most suitable candidate for the role of such a "channeling" compound is $\mathrm{Mo}_{8} \mathrm{O}_{23}$ because in this case the value of $T_{c 1}$ almost coincides with the above reported experimentally measured $T_{0}$. It should be noted that formation of other compounds of molybdenum (e.g., pyrochlores, bronzes, sulfides, or chalcogenide glasses), exhibiting MITs with similar order of magnitude transition temperatures $[14-18,20]$ is impossible because of the lack of the necessary chemical elements (i.e., rare earths, alkali metals or hydrogen, sulfur, and tellurium) in the phase composition of both the oxide film and the metal electrodes ( $\mathrm{Au}, \mathrm{Ta}, \mathrm{Mo})$.

\section{Conclusion}

The results presented in this paper on switching from the high- to the low-resistance state with an S-type NDR in molybdenum oxides obtained by both thermal vacuum deposition and electrochemical anodic oxidation show that the switching effect is due to, apparently, an insulator-tometal phase transition in the switching channel consisting, completely or partly, of the lower oxide $\mathrm{Mo}_{8} \mathrm{O}_{23}$, formed in the initial film during the process of electroforming. The current flowing through the channel heats it up to the MIT temperature $T_{c 1}=315 \mathrm{~K}[12]$ and the structure switches into the metallic low-resistance state.

Thus, the channels, consisting of this lower oxide, are formed in initial Mo oxide films during preliminary electroforming. Electrical forming results in the changes in oxygen stoichiometry conditioned by the ionic processes in electric fields close to the MOM structure breakdown field. This picture is quite similar to what is observed in many other TMOs exhibiting the insulator-to-metal transitions $[15,22$, 27, 33-35]. The results obtained concerning the threshold switching in thin-film MOM structures with molybdenum oxides indicate the possibility of application of this material in oxide electronics as micro- and nanostructured switching elements and other electronic devices.

\section{Conflict of Interests}

The authors declare that there is no conflict of interests regarding the publication of this paper.

\section{Acknowledgments}

This work was supported by the Strategic Development Program of Petrozavodsk State University (2012-2016) and the RF Ministry of Education and Science as a base part of state Program no. 2014/154 in the scientific field, Project no. 1704, and state Program no. 3.757.2014/K.

\section{References}

[1] P. A. Cox, Transition Metal Oxides. An Introduction to their Electronic Structure and Properties, Clarendon Press, Oxford, UK, 1992.

[2] L. Mai, F. Yang, Y. Zhao et al., "Molybdenum oxide nanowires: synthesis \& properties," Materials Today, vol. 14, no. 7-8, pp. 346-353, 2011.

[3] M. C. Rao, K. Ravindranadh, A. Kasturi, and M. S. Shekhawat, "Structural stoichiometry and phase transitions of $\mathrm{MoO}_{3}$ thin films for solid state microbatteries," Research Journal of Recent Sciences, vol. 2, no. 4, pp. 67-73, 2013.

[4] V. P. Malinenko, A. L. Pergament, and A. O. Gorbakov, "Electrical instabilities in thin film structures on the basis of molybdenum oxides," Proceedings of Petrozavodsk State University, no. 2(139), pp. 100-106, 2014.

[5] R. L. Smith and G. S. Rohrer, "Scanning probe microscopy of cleaved molybdates: $\alpha-\mathrm{MoO}_{3}(010), \quad \mathrm{Mo}_{18} \mathrm{O}_{52}(100)$, $\mathrm{Mo}_{8} \mathrm{O}_{23}(010)$, and $\eta-\mathrm{Mo}_{4} \mathrm{O}_{11}(100)$," Journal of Solid State Chemistry, vol. 124, no. 1, pp. 104-115, 1996.

[6] D. O. Scanlon, G. W. Watson, D. J. Payne, G. R. Atkinson, R. G. Egdell, and D. S. L. Law, "Theoretical and experimental study of the electronic structures of $\mathrm{MoO}_{3}$ and $\mathrm{MoO}_{2}$," The Journal of Physical Chemistry C, vol. 114, pp. 4636-4645, 2010.

[7] M. Arita, H. Kaji, T. Fujii, and Y. Takahashi, "Resistance switching properties of molybdenum oxide films," Thin Solid Films, vol. 520, no. 14, pp. 4762-4767, 2012.

[8] M. Hasan, "A materials approach to resistive switching memory oxides," Journal of Semiconductor Technology and Science, vol. 8, no. 1, pp. 66-79, 2008.

[9] A. I. Gavrilyuk and N. A. Sekushin, Electrochromism and Photochromism in Oxides of Tungsten and Molybdenum, Nauka, Leningrad, Russia, 1990, (Russian).

[10] S.-Y. Lin, C.-M. Wang, K.-S. Kao, Y.-C. Chen, and C.-C. Liu, "Electrochromic properties of $\mathrm{MoO}_{3}$ thin films derived by a solgel process," Journal of Sol-Gel Science and Technology, vol. 53, no. 1, pp. 51-58, 2010.

[11] Y.-S. Lin, T.-H. Tsai, W.-H. Lu, and B.-S. Shie, "Lithium electrochromic properties of atmospheric pressure plasma jetsynthesized tungsten/molybdenum-mixed oxide films for flexible electrochromic device," Ionics, vol. 20, no. 8, pp. 1163-1174, 2014.

[12] H. Fujishita, M. Sato, S. M. Shapiro, and S. Hoshino, "Inelastic neutron scattering of the low-dimensional conductors $\left(\mathrm{TaSe}_{4}\right)_{2} \mathrm{I}$ and $\mathrm{Mo}_{8} \mathrm{O}_{23}$," Physica $B+C$, vol. 143, no. 1-3, pp. 201-203, 1986.

[13] T. Sato, T. Dobashi, H. Komatsu, T. Takahashi, and M. Koyano, "Electronic structure of $\eta-\mathrm{Mo}_{4} \mathrm{O}_{11}$ studied by high-resolution angle-resolved photoemission spectroscopy," Journal of Electron Spectroscopy and Related Phenomena, vol. 144-147, pp. 549-552, 2005.

[14] Y. Motome and N. Furukawa, "Orbital degeneracy and Mott transition in Mo pyrochlore oxides," Journal of Physics: Conference Series, vol. 320, Article ID 012060, 2011. 
[15] A. Maeda, T. Furuyama, and S. Tanaka, "Threshold-field behavior and switching in $\mathrm{K}_{0.3} \mathrm{MoO}_{3}$," Solid State Communications, vol. 55, no. 11, pp. 951-955, 1985.

[16] Y. C. Lin, D. O. Dumcenco, Y. S. Huang, and K. Suenaga, "Atomic mechanism of the semiconducting-to-metallic phase transition in single-layered $\mathrm{MoS}_{2}$," Nature Nanotechnology, vol. 9, no. 5, pp. 391-396, 2014.

[17] X.-Y. Xu, Z.-Y. Yin, C.-X. Xu, J. Dai, and J.-G. Hu, "Resistive switching memories in $\mathrm{MoS}_{2}$ nanosphere assemblies," Applied Physics Letters, vol. 104, no. 3, Article ID 033504, 2014.

[18] R. Rousseau, E. Canadell, P. Alemany, D. H. Galván, and R. Hoffmann, "Origin of the metal-to-insulator transition in $\mathrm{H}_{0.33} \mathrm{MoO}_{3}$," Inorganic Chemistry, vol. 36, no. 21, pp. 4627-4632, 1997.

[19] E. Canadell and M.-H. Whangbo, "Band electronic structure study of the structural modulation in the Magnéli phase $\mathrm{Mo}_{8} \mathrm{O}_{23}$, Inorganic Chemistry, vol. 29, no. 12, pp. 2256-2260, 1990.

[20] S. Mukherjee, S. Karmakar, H. Sakata, and B. K. Chaudhuri, "Low-temperature metallic behavior of amorphous $\mathrm{MoO}_{3}$ $\mathrm{TeO}_{2}$ thin films," Journal of Applied Physics, vol. 97, no. 12, Article ID 123707, 2005.

[21] A. L. Pergament, P. A. Boldin, V. V. Kolchigin, and T. G. Stefanovich, "Bistable switching and memory effects in transition metal oxides," Proceedings of Petrozavodsk State University, no. 8(121), pp. 118-120, 2011.

[22] A. Pergament, G. Stefanovich, A. Velichko, V. Putrolainen, T. Kundozerova, and T. Stefanovich, "Novel hypostasis of old materials in oxide electronics: metal oxides for resistive random access memory applications," in Research in Novel Materials, R. Islam, Ed., pp. 77-104, Nova Science Publiher, 2013.

[23] S. Balatti, S. Larentis, D. C. Gilmer, and D. Ielmini, "Multiple memory states in resistive switching devices through controlled size and orientation of the conductive filament," Advanced Materials, vol. 25, no. 10, pp. 1474-1478, 2013.

[24] D. B. Strukov and R. S. Williams, "An ionic bottle for highspeed, long-retention memristive devices," Applied Physics A, vol. 102, no. 4, pp. 1033-1036, 2011

[25] R. Xie, C. T. Bui, B. Varghese et al., "An electrically tuned solid-state thermal memory based on metal-insulator transition of single-crystalline $\mathrm{VO}_{2}$ nanobeams," Advanced Functional Materials, vol. 21, no. 9, pp. 1602-1607, 2011.

[26] A. Mottaghizadeh, Q. Yu, P. L. Lang, A. Zimmers, and H. Aubin, "Metal oxide resistive switching: evolution of the density of states across the metal-insulator transition," Physical Review Letters, vol. 112, no. 6, Article ID 066803, 2014.

[27] F. A. Chudnovskii, L. L. Odynets, A. L. Pergament, and G. B. Stefanovich, "Electroforming and switching in oxides of transition metals: the role of metal-insulator transition in the switching mechanism," Journal of Solid State Chemistry, vol. 122, no. 1, pp. 95-99, 1996.

[28] S. A. Gavrilov and A. N. Belov, Electrochemical Processes in Technology of Micro- and Nanoelectronics, Higher School, Moscow, Russia, 2009 (Russian).

[29] M. R. Arora and R. Kelly, "The structure and stoichiometry of anodic films on V, Nb, Ta, Mo and W,' Journal of Materials Science, vol. 12, no. 8, pp. 1673-1684, 1977.

[30] C. M. Daly and R. G. Keil, "On the anodic oxidation of molybdenum," Journal of the Electrochemical Society, vol. 122, no. 3, pp. 350-353, 1975.
[31] A. L. Pergament and G. B. Stefanovich, "Phase composition of anodic oxide films on transition metals: a thermodynamic approach," Thin Solid Films, vol. 322, no. 1-2, pp. 33-36, 1998.

[32] R. Swanepoel, "Determination of the thickness and optical constants of amorphous silicon," Journal of Physics E: Scientific Instruments, vol. 16, no. 12, pp. 1214-1221, 1983.

[33] A. L. Pergament, V. P. Malinenko, L. A. Aleshina, and V. V. Kolchigin, "Metal-insulator phase transition and electrical switching in manganese dioxide," Physics of the Solid State, vol. 54, no. 12, pp. 2486-2490, 2012.

[34] A. L. Pergament, V. P. Malinenko, O. I. Tulubaeva, and L. A. Aleshina, "Electroforming and switching effects in yttrium oxide," Physica Status Solidi (A) Applied Research, vol. 201, no. 7, pp. 1543-1550, 2004.

[35] A. L. Pergament, P. P. Boriskov, A. A. Velichko, and N. A. Kuldin, "Switching effect and the metalinsulator transition in electric field," Journal of Physics and Chemistry of Solids, vol. 71, no. 6, pp. 874-879, 2010. 

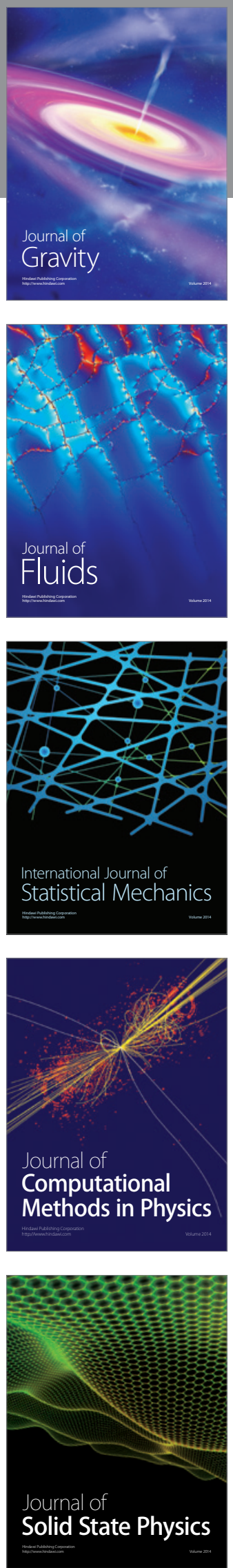

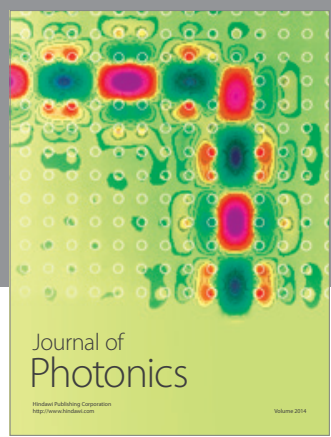

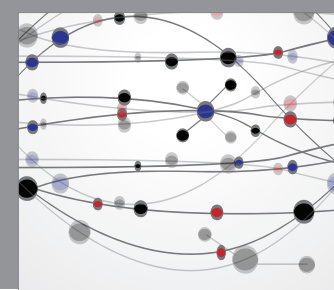

The Scientific World Journal

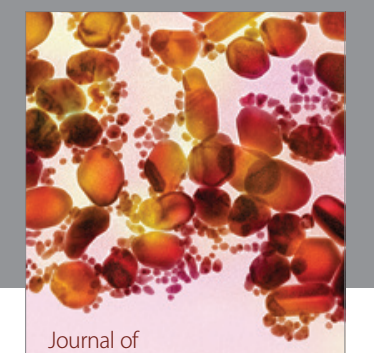

Soft Matter
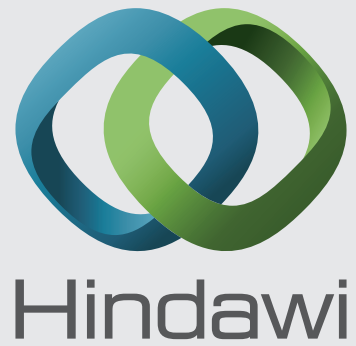

Submit your manuscripts at

http://www.hindawi.com
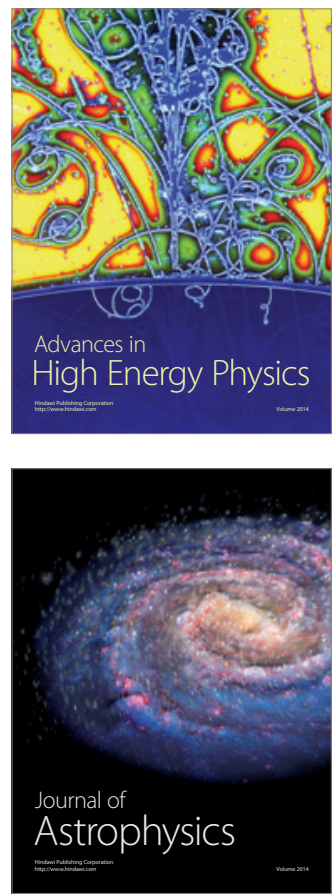
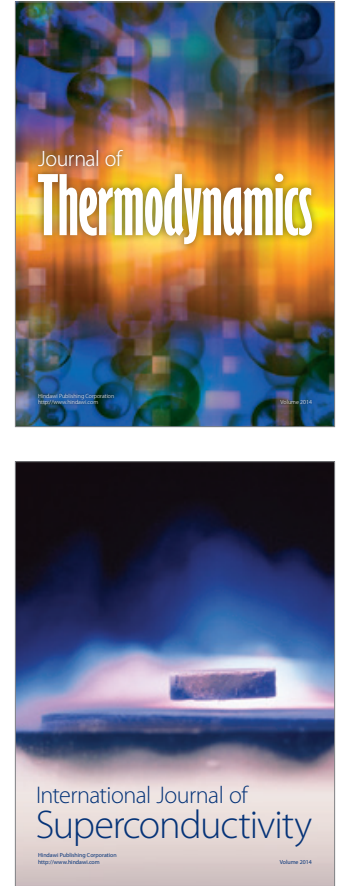
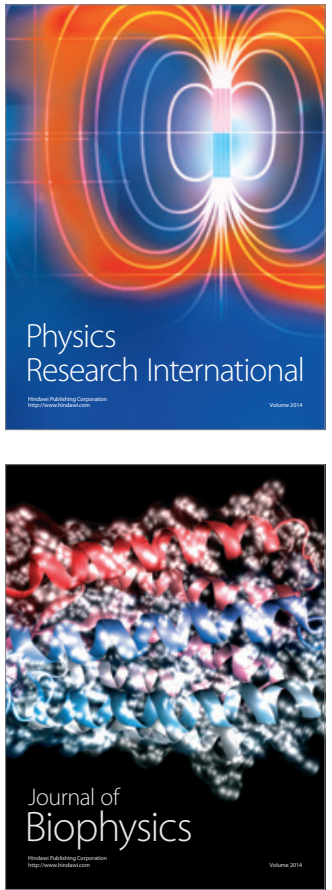
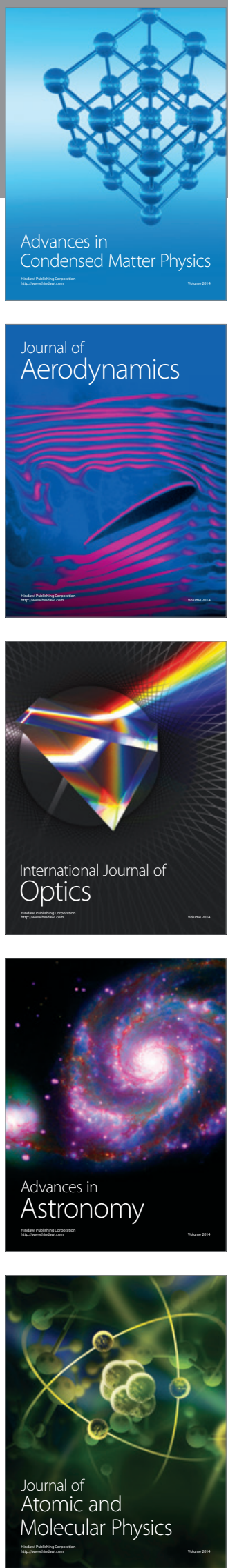